\section{OP63 THE EFFECTS OF THE 2007-9 FINANCIAL CRISIS ON MENTAL HEALTH IN THE UK: A LONGITUDINAL ANALYSIS OF NON-SUICIDE MENTAL HEALTH TRENDS}

${ }^{1} \mathrm{~B}$ Amies*, ${ }^{2} \mathrm{~L}$ Munford, ${ }^{2} \mathrm{M}$ Sutton. ${ }^{1}$ Centre for Primary Care, University of Manchester, Manchester, UK; ${ }^{2}$ Manchester Centre for Health Economics, University of Manchester, Manchester, UK

\subsection{6/jech-2017-SSMAbstracts.62}

Background Existing research using area-level data has identified big increases in suicides in the UK and elsewhere through the financial crisis of 2007-09. Areas with larger increases in unemployment tended to have greater increases in suicide rates. The evidence base in this area has two particular weaknesses that this study aims to address. Firstly, this study uses individual-level data rather than aggregated area data, and secondly it examines non-suicide mental health trends rather than suicide rates.

Methods A nationally-representative sample of 12816 individuals from the British Household Panel Survey in England from 2000-2013. Data were not collected for 2009. Fixed effects models were used to examine the data for associations between 12-item General Health Questionnaire score (measuring mental health) and demographic features, employment status and equivalised household income on the unweighted sample. Dummy variables for 2008-2010 (the period from the start of recession up to the start of government budget cuts) and 2011-13 (from the start of budget cuts) separated out the influence of changing economic conditions. Lagged and advanced variables were used to explore associations between mental health and the loss of income or employment through time.

Results Poor mental health peaked in 2008, before the peak in unemployment in 2010. Women and poorer people had significantly worse mental health throughout $(\mathrm{p}<0.000$, beta $1.24(95 \%$ confidence interval 1.08-1.40), $\mathrm{p}<0.000$, beta $0.129(-0.192--0.065)$ respectively). Unemployment was significantly associated with worse mental health $(\mathrm{p}<0.000$, beta $1.84(1.52-2.16))$. The association between mental health and income was weaker, with the most persistent effects for the period 2008-2010 ( $\mathrm{p}=0.000$, beta $-0.64 \quad(-0.88-\quad-0.40))$. Worse mental health appeared to preced job or income loss $(\mathrm{p}=0.028$, beta $0.46(0.049-0.88) ; \mathrm{p}=0.011$, beta 0.23 (0.051-0.40), respectively) but mental health did not deteriorate following job-loss, while increasing income appeared to compromise mental health $(\mathrm{p}=0.006$, beta $-0.24 \quad(-0.42-$ $-0.069)$ ). Weighted sensitivity analysis confirmed a persistent association between unemployment and mental health but significance was lost between income and mental health.

Conclusion Non-suicide mental health deteriorated during the 2007-2009 financial crisis. It appears that worse mental health preceded job loss or income loss. A possible explanation could be that poor mental health conferred weakness in the job market. There are a number of inferences to the research area, for example that a strong social safetynet may protect mental health (by maintaining income) and that in-work mental health support may benefit poorer workers.

\section{OP64 DO RELIGIOUS PRACTICES AND BELIEFS MODERATE THE ASSOCIATION BETWEEN STRESSFUL LIFE EVENTS AND SUBSEQUENT MENTAL HEALTH AND WELLBEING?}

${ }^{1} \mathrm{~A}$ Kaushal*, ${ }^{2} \mathrm{D}$ Cadar, ${ }^{1} \mathrm{M}$ Stafford, ${ }^{1} \mathrm{M}$ Richards. ${ }^{1} \mathrm{M} \mathrm{C} C$ Unit for Lifelong Health and Ageing, University College London, UK; ${ }^{2}$ Research Department of Behavioural Science and Health, University College London, UK

\subsection{6/jech-2017-SSMAbstracts.63}

Background Religion may provide coping strategies for stressful life events (SLEs). Aspects of religious practices and beliefs, e.g. provision of meaning in life, interpretation of difficult events and supportive social contact can all contribute to successful coping strategies. The aim of this project was to investigate if SLEs are associated with mental health and wellbeing in early old age and if religious practices and beliefs moderate these associations.

Methods Participants were study members from the MRC National Survey of Health and Development (1946 British birth cohort) who participated in data collection at age 68-69 $(n=2148)$. Mental health and wellbeing were measured using the 28-item General Health Questionnaire and the WarwickEdinburgh Mental Wellbeing Scale. SLEs were measured using a checklist of events on five occasions from age 26 to 60-64. Religious practices and beliefs were ascertained from age 11 to 68-69 and included upbringing, beliefs and attendance. Initial analyses investigated associations between SLEs, and mental health and wellbeing. This was followed by analyses to determine if religious practices and beliefs were more common in people who had good mental health or high wellbeing despite experiencing a high number of SLEs compared to those with fewer SLEs and poor mental health or wellbeing. Analyses were conducted using linear and logistic regression models adjusted for gender and education.

Results Experiencing SLEs was associated with lower wellbeing $(\beta=-0.31, C I=-0.46,-0.16)$ and worse mental health at age 68-69 $(\beta=0.02, C I=0.02,0.03)$. High wellbeing at age 68 despite a substantial number of SLEs was associated with frequent religious attendance (for men only) from age 36 to 60 $64 \quad(\mathrm{OR}=2.60, \quad \mathrm{CI}=1.10,6.14), \quad$ religious importance $(\mathrm{OR}=1.83, \mathrm{CI}=1.13,2.97)$ and meaning in life provided by religion $(\mathrm{OR}=2.07, \mathrm{CI}=1.31,3.29)$. There were no differences in religious practices and beliefs between study members with good mental health and a high number of SLEs compared to those with fewer SLEs and poor mental health.

Conclusion SLEs across the life course were associated with poor mental health and wellbeing in early old age. We also found evidence that religious practices and beliefs moderate the effect of SLEs on wellbeing but not mental health, and in particular that religious attendance is beneficial for men and not women. Future work will investigate if SLEs are associated with changes in religious attendance, mental health and wellbeing, and if this varies by different types of SLEs, e.g. personal and interpersonal, health and work-related events. 


\section{Behaviours 2}

\section{OP65 A SYSTEMATIC REVIEW OF THE PREVALENCE OF SMOKING IN HEALTHCARE STUDENTS}

AE Granville*, T McKeever, R Murray, K Nilan. Epidemiology and Public Health, University of Nottingham, Nottingham, UK

\subsection{6/jech-2017-SSMAbstracts.64}

Background Smoking continues to pose a huge cost to an individuals' health and the healthcare economy. Healthcare professionals are known to have an authoritative influence over patients and are well placed to promote abstinence from tobacco. Indeed, Articles 12 and 14 of the Framework Convention on Tobacco Control (FCTC) make several recommendations concerning smoking behaviour and cessation training amongst healthcare professionals. The current study estimates the prevalence of smoking in healthcare students, healthcare professionals of the future, across the six World Health Organisation regions.

Methods Five databases (Medline, Embase, CINAHL, CAB abstracts, LILACS and the WHO Global Healthcare Professional Survey database) were searched to identify studies including any profession of healthcare students. Studies were published between January 2000 and March 2016, and no restrictions were placed on language of publication. Titles, abstracts and full texts were checked for eligibility independently by two authors and the quality of the included studies was assessed. Pooled prevalence with 95\% confidence intervals (CI) were estimated using random effect models, with heterogeneity quantified using $\mathrm{I}^{2}$.

Results 417 papers were included: 214 studies and 203 Global Health Professional student surveys. Healthcare professions included medicine, nursing, dentistry, pharmacy, and mixed groups. The prevalence of smoking across all healthcare students was 19\% (95\% CI 17\%-21\%, I ${ }^{2}$ 99.98). Subgroup analysis by year shows the prevalence of smoking in healthcare students appears to be increasing; from 16\% (95\% CI 16\%$\left.17 \%, I^{2} 99.23\right)$ up to and including 2010 to $19 \%$ (95\% CI 6\%-31\%, I $\mathrm{I}^{2}$ 99.99) between 2011 and 2016. Pooled estimated smoking prevalence within the WHO regions for medical students ranged from $10 \%$ to $25 \%$, and nursing students from $0 \%$ to $30 \%$. Estimates for both were highest in Europe and lowest in Africa.

Conclusion Smoking prevalence among healthcare students varies widely across professions and WHO regions, however remains worryingly high in light of the key role healthcare professionals play in tackling the global smoking epidemic. In order to continue to make progress with implementation of the FCTC, urgent efforts need to be made to reduce smoking behaviour amongst healthcare students, which will ultimately contribute to the reduction of smoking prevalence amongst their patients.

\section{OP66 MOTIVATION TO QUIT SMOKING AND CHANGES IN CIGARETTE CONSUMPTION, AMONG SMOKERS WHO USE E-CIGARETTES, FINDINGS FROM THE HEALTH SURVEY FOR ENGLAND}

L Ng Fat*', S Scholes, JS Mindell. Epidemiology and Public Health, UCL, London, UK
Background The majority of people who use e-cigarettes are dual users with tobacco cigarettes. E-cigarettes may aid smokers with their quit attempts and reduce cigarette consumption or reinforce nicotine addiction. This study explores the motivations for current and previous use of e-cigarettes, and whether use is associated with reporting lower or higher cigarette consumption than a year ago. It makes comparisons with other traditional nicotine delivery products (NDPs).

Methods This study uses a sample of current smokers aged 16 $+(n=3,039)$ from the nationally representative, cross-sectional Health Survey for England, HSE2013-2014, (HSE2015 data will be included when archived). Firstly, multinomial logistic regression models were conducted on the odds of a) Never use of e-cigarettes versus b) Current use of e-cigarettes c) Previous (not current) use of e-cigarettes, and key exposure included the intentions to quit smoking scale (No intention/ Pre-contemplation/Contemplation/Preparation (within next 3 months)). Secondly multinomial logistic regression was carried out on the odds of reporting smoking a) the same number of cigarettes versus b) more c) fewer than a year ago. Models were repeated using never, current and previous use of other NDPs. All models adjusted for sex, age-group, highest qualification and cigarette consumption. Analyses were conducted using Stata.

Results 12\% were current users, and 20\% previous users of ecigarettes. Compared with never use of e-cigarettes, no association was found with age and current use, while being younger was associated with previous use $(45-54 \mathrm{v}$. 1634 years, $\mathrm{OR}=0.68$ [95\%CI 0.46-0.79]). Conversely, current and previous users of NDPs were more likely to be older than never users of NDPs (45-54 years 2.07 [1.29-3.30]). Quit intentions had a dose response relationship with the odds of current e-cigarette use (e.g. Preparation versus No intention (3.14 [2.24-4.42]); for previous e-cigarette users the magnitude was smaller (1.39 [1.04-1.87]). However, 'Preparation' had stronger associations with other NDPs, for both current (8.93 [5.54-14.40]), and previous use (3.18 [2.47-4.09]). Being a current user of e-cigarettes (1.77 [1.36-3.20]) or other NDPs (1.72 [1.19-2.50]) increased the odds of reporting smoking fewer cigarettes than the previous year; previous use was not significant. E-cigarette use was not associated with reporting smoking more than the previous year, however current use of NDPs was (1.84 [1.13-3.01]).

Conclusion Current or previous e-cigarette use is unlikely to increase consumption of cigarettes compared with a year ago, but smokers who used them had weaker intentions to quit smoking than smokers who used other NDPs. Longitudinal research is needed to track changes in consumption involving duration of e-cigarette use to further verify findings.

\section{OP67 ACTIVITY LEVELS IN MOTHERS AND CHILDREN DURING THE TRANSITION TO PRIMARY SCHOOL: FINDINGS FROM THE SOUTHAMPTON WOMEN'S SURVEY}

${ }^{1} \mathrm{KR}$ Hesketh*, 'S Brage, ${ }^{1,2} \mathrm{U}$ Ekelund, ${ }^{3,4,5} \mathrm{C}$ Cooper, ${ }^{3,4} \mathrm{~K}$ Godfrey, ${ }^{3,4} \mathrm{NC}$ Harvey, ${ }^{3,4} \mathrm{H}$ Inskip, ${ }^{3,4}{ }^{1}$ S Robinson, ${ }^{1} \mathrm{EM}$ van Sluijs. ${ }^{1}$ CEDAR and MRC Epidemiology Unit, University of Cambridge, Cambridge, UK; ${ }^{2}$ Department of Sport Medicine, Norwegian School of Sport Sciences, Oslo, Norway: ${ }^{3}$ MRC Lifecourse Epidemiology Unit, University of Southampton, Southampton, UK; ${ }^{4}$ NIHR Southampton Biomedical Research Centre, University of Southampton and, University of Southampton and University Hospital Southampton NHS Foundation, Southampton, UK; ${ }^{5}$ NIHR Musculoskeletal Biomedical Research Centre, University of Oxford, Oxford, UK 